\title{
Plant Leaf Imaging using Time of Flight Camera under Sunlight, Shadow and Room Conditions
}

\author{
Wajahat Kazmi \\ Department of Architecture, Design and Media Technology \\ Aalborg University, Denmark \\ Email: wajahat@create.aau.dk
}

\author{
Sergi Foix and Guillem Alenyà \\ Institut de Robòtica i Informàtica Industrial \\ CSIC-UPC, Barcelona, Spain \\ Email: sfoix@iri.upc.edu, galenya@iri.upc.edu
}

\begin{abstract}
In this article, we analyze the effects of ambient light on Time of Flight (ToF) depth imaging for a plant's leaf in sunlight, shadow and room conditions. ToF imaging is sensitive to ambient light and we try to find the best possible integration times (IT) for each condition. This is important in order to optimize camera calibration. Our analysis is based on several statistical metrics estimated from the ToF data. We explain the estimation of the metrics and propose a method of predicting the deteriorating behavior of the data in each condition using camera flags. Finally, we also propose a method to improve the quality of a ToF image taken in a mixed condition having different ambient light exposures.
\end{abstract}

\section{INTRODUCTION}

Depth imaging for indoor applications is becoming more popular especially with the advent of RGBD cameras. Tasks in which better depth resolution and ambient light cancellation are desirable, Time of Flight (ToF) cameras are still a more preferred but relatively expensive solution. Various brands of ToF cameras are available in the market but their performance, generally, is compromised under strong sunlight conditions. ToF cameras are active sensors having Near InfraRed (NIR) light emitters $(850 \mathrm{~nm})$ and scene depth is estimated from time required for the light to reflect back to the sensor. The sensor is fairly low resolution (max: 200x200 at present) and low signal-to-noise ratio (SNR). A good reference for explaining the working principal is Lange and Seitz [1] and for noise characteristics of state-of-the-art ToF cameras are Foix et al. [2] and Mufti and Mahony [3]. For an ambitious reader, a detailed study with derivations of mathematical equations can be found in the $\mathrm{PhD}$ thesis of Lange [4]. In short, ToF cameras typically return registered depth, amplitude and intensity images of the same size.

Our domain of research is agriculture, where it is important to monitor and measure the morphological properties of plants (such as size and structure) in order to quantify plant density, vigor and identify the species. Digital imaging is an important tool for this task but mere 2D imaging is insufficient to cope with overlapping and complicated plant

This work is supported by the Danish Council for Strategic Research under project ASETA (www.aseta.dk) grant no. 09-067027, the Spanish Ministry of Science and Innovation under projects PAU + DPI2011-27510, the EU project GARNICS (FP7-247947) and the Catalan Research Commission (SGR-00155). W. Kazmi is funded by ASETA and S. Foix is supported by PhD fellowship from CSIC's JAE program
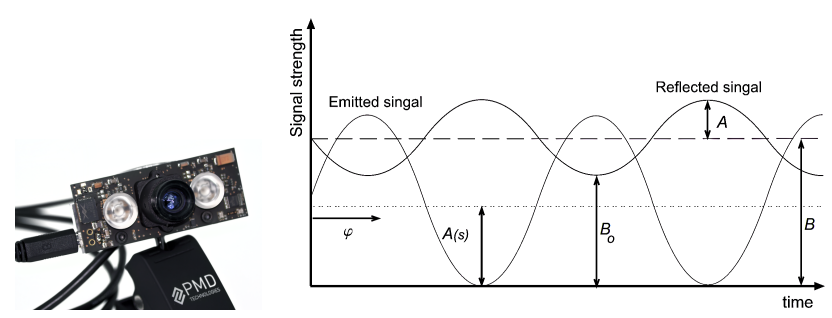

(a) PMDTec CamBoard (b) Influence of background illumination on ToF signal, Mufti and Mahony [3]

Fig. 1. PMD CamBoard and ToF working principle

canopies. Depth sensing is hence important, especially in modern phenotyping facilities which use robotic manipulators to grasp desired parts of the plant. This task demands highly precise and accurate depth data. Stereovision vision suffers from the correspondence problem and efficiency bottlenecks when working at close ranges for low texture leaf surfaces. ToF cameras provide high frame rate depth data (more than $30 \mathrm{fps}$ ) without correspondence issues which is proving vital in this regard. Therefore, ToF imaging is finding a greater utility in agricultural projects (such as ASETA [5] and GARNICS [6]). It is hence pertinent to review important work done in agriculture using ToF technology.

\section{A. ToF imaging in agriculture}

Feasibility of ToF cameras for plant analysis has been done by Klose et al. [7] and Kraft et al. [8] but they fail to address some important aspects such as integration time (IT: time for which sensor integrates the returned signal) without which ToF data evaluation becomes somewhat meaningless. Still, these works found $\mathrm{ToF}$ a potential candidate for plant phenotyping. Alenya et al. [6] also investigated ToF potential for plant phenotyping. Their focus stays limited to indoor environments by combining ToF with RGB images. In field operations, both light and wind pose a great challenge. Nakarmi and Tang [9] used SR4000 ToF camera for inter-plant distance measurement in corn fields. Their camera assembly covered both the camera and the view from sunlight and wind. To the best of authors' knowledge, depth imaging for plants have not been compared under natural and room illumination settings.

Although ToF light is modulated on a carrier wave typically having frequency in the range of $15-30 \mathrm{Mhz}$, its reflection, 


\begin{tabular}{|l|c|l|}
\hline Flag & Identifier (Hex) & Description \\
\hline \hline Invalid & 0x00000001u & $\begin{array}{l}\text { Depth value unreliable } \\
\text { (amplitude too high or too low) }\end{array}$ \\
\hline Inconsistent & $0 \mathrm{x} 00000004 \mathrm{u}$ & $\begin{array}{l}\text { Raw data inconsistent } \\
\text { (motion artifacts, saturation etc) }\end{array}$ \\
\hline Low Signal & $0 \mathrm{x} 00000008 \mathrm{u}$ & $\begin{array}{l}\text { Not enough signal strength to } \\
\text { measure depth accurately }\end{array}$ \\
\hline SBI Active & $0 \mathrm{x} 00000010 \mathrm{u}$ & $\begin{array}{l}\text { Suppression of background } \\
\text { illumination is active }\end{array}$ \\
\hline
\end{tabular}

TABLE I

CAMBOARD FLAGS transmission and absorption largely depends on the NIR frequency in it. As this light is not in the visible range, it is necessary to carry out a brief survey on optical characteristics of NIR rays on plant leaves.

\section{B. Leaf optical characteristics in NIR spectrum}

Light interaction on plant canopy and leaf surface, which includes both the photosynthetically active radiation (PAR) as well as infrared radiation (IR), has been studied for many decades. From the surface of a leaf, some part of the incident light is absorbed, some is reflected and the rest is transmitted. For leaf surface analysis, reflectance ought to be higher. Woolley [10] found that both the reflectance and transmittance for soyabean and maize leaves were high in NIR region as compared to visible spectrum, since the plants absorb significant amount of incident visible light. Similar results were achieved by reflectance-transmittance model proposed by Jacquemoud and Baret [11] which showed almost $51 \%$ reflectance, $45 \%$ transmittance and $4 \%$ absorption for green soyabean leaves in the NIR region. This reflectance is low but in their findings on a frequency scale from $400 \mathrm{~nm}$ to $2500 \mathrm{~nm}$, which also includes most of the visible spectrum, the only region having highest reflectance and lowest possible transmittance and absorption is the NIR region between 750 $\mathrm{nm}$ and $1000 \mathrm{~nm}$. ToF cameras operating at $850 \mathrm{~nm}$ are right in the ball park, making them quite feasible for leaf imaging.

However, one major problem is the saturation of ToF cameras under sunlight as mentioned in the manufacturer documentation. Only PMD cameras are claimed to work under sunlight, but not out-of-the-box as IT has to be adjusted for a given ambient illumination and object distance. As discussed by May et al. [12], IT affects the amplitude and intensity of the data. They also proposed a method to find best integration times avoiding both saturation and low signal for ToF cameras using amplitude and intensity variation with IT. With new cameras, the manufacturer's application programming interface (API) returns either a confidence matrix (SwissRanger [13]) or flags (PMD [14]) which comment on the behavior of every pixel during image acquisition, which we intend to use.

In this article, we provide a comprehensive study of the response of a PMD CamBoard camera under sunlight, shadow and room conditions and we propose a criteria to select different camera integration times based on the internal error flags and the amplitude values. The challenge is to find a suitable IT for each of the three conditions because a different calibration has to be applied for each IT and calibration is a costly process. Additionally, we propose a method to obtain an optimal image when sunlight and shadow are both present at the same time.

The breakdown of this paper is as follows: In Sec. II, we briefly describe the hardware. Mathematical relationship between IT and amplitude is discussed in Sec. III, followed by experimental setup in Sec. IV. In Sec. V, metrics for evaluation of data are defined. Data analysis is done in Sec. VI. Sec. VII concludes the paper.

\section{PMDTEC CAMBoard: Specifications}

The choice of CamBoard is due to its small size and low power consumption (USB powered). It is a webcam style ToF camera (Fig. 1(a)) by PMD Technologies. Image sensor size is $200 \times 200$ pixels. It returns a depth image, 3D data, registered amplitude and intensity images and a limited set of flags per pixel. CamBoard works on the standard Lock-in Time of flight principle [2]. NIR light is typically modulated at a $20 \mathrm{Mhz}$ carrier although it can be changed. Continuous Wave $(\mathrm{CW})$ modulation is employed and phase difference is used to estimate depth rather than directly measuring the turn around time of the signal.

\section{A. Depth}

Depth image returned by CamBoard contains the $Z$ coordinate of the object in view in meters. The data is signed double. The higher are the depth values, the farther is the object from the camera. Integrity of the depth data is judged by amplitude and flags.

\section{B. Amplitude}

The amplitude has no specific units. It represents the amount of NIR light reflected from the scene. Higher amplitude means more confidence in the measurement. But a very high amplitude leads to saturation which is an indication that no more photons can be accommodated by the pixels, thus producing unreliable depth measurements.

\section{Intensity}

CamBoard returns a typical 8 bit intensity image of the same size as depth image.

\section{Flags}

The flag matrix of CamBoard has a 32 bit flag value for every pixel. If set, the reason could be one or more of the flags in Tab. I. Sometimes, with Invalid flag, other bits provide information for the possible reason(s) of invalidity.

\section{EFFECT OF AMPLITUDE AND IT ON DEPTH PRECISION}

Depth precision is directly related to the amplitude and is given by [1]:

$$
\Delta L=\frac{L}{2 \pi} . \Delta \varphi=\frac{c}{4 \pi f_{\text {mod }}} \frac{\sqrt{B}}{\sqrt{2} A}
$$


where $\Delta L$ is the depth precision (or range resolution) due to photon-shot noise (quantum noise), $B$ (intensity) is the offset to account for background illumination $B_{0}$ and mean amplitude $A$ of the returned active illumination [3]:

$$
B=B_{0}+A
$$

and $L$ is the maximum depth:

$$
L=\frac{c}{2 f_{\text {mod }}}
$$

here $c$ is the speed of light and $f_{\text {mod }}$ is the modulation frequency (20 Mhz for CamBoard). Details of the derivation can be found in the thesis of Lange [4] chapter 4. According to Mufti and Mahony [3], ToF signal to noise ratio (SNR) is given by:

$$
S N R=\frac{\sqrt{2} A}{\sqrt{B}}
$$

and therefore Eq. 1 can be reduced to:

$$
\Delta L=\frac{c}{4 \pi f_{\text {mod }}} \frac{1}{S N R}=\sigma_{L}
$$

Photon shot noise is represented by Poisson distribution of the process of arrival of photons at the sensor which in case of very large number of photons (as in ToF cameras) can be approximated by Gaussian distribution with the standard deviation $\sigma_{L}$ of the range measurement [3]. According to Eq. 1 and also from general observation, low amplitude decreases SNR and makes depth invalid. Very high amplitude, on the other hand, saturates the sensor producing incorrect depth estimate. It seems convenient then to have an upper threshold for amplitude. But amplitude is directly controlled through IT for a given distance, due to which IT plays a key role in precise depth estimation. Strong background illumination $B_{0}$ (such as sunlight) increases $B$ and hence worsens this situation. So in order to reduce its effect, IT must be reduced which in turn decreases $A$. $B$ also includes $A$, but due to square root dependence of $\sigma_{L}$ on $B$, an increase in $A$ results in an overall increase of precision. It is therefore obvious, that an optimum IT is a best compromise between $\sigma_{L}, A$ and $B$ and no single value of IT can be optimal for different ambient illumination settings. This fact makes use of ToF cameras more challenging as for more accurate measurements, ToF camera must be calibrated for a specific IT which means that an optimal calibration for outdoor conditions will not be suitable for indoor applications and if used, will have integration time related errors [2].

For CamBoard, we have found the flag matrix obtained through the manufacturer's API very useful in this regard. We use the flag matrix to find pixels marked Invalid due to low signal amplitude or inconsistency as a result of strong scintillation or multiple depth values which could probably be caused either by multiple reflections, rapid motion or saturation. PMD cameras have an on-board circuitry for suppression of background illumination (SBI) along with an associated flag. Our analysis will also test its performance under sunlight.
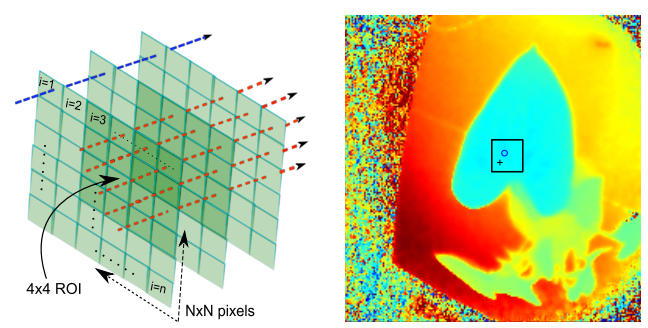

(a) Mean and Std.Dev. (b) 20x20 ROI on leaf imacross frames with a sample age used for analysis $4 \times 4$ ROI

Fig. 2. Data Interpretation

\section{EXPERIMENTAL SETUP}

The object in view was a plant leaf, Anthurium Andraeanum. Plant was grown in pot and the camera-to-leaf distance was between $30-35 \mathrm{~cm}$. Imaging was performed under three ambient illumination conditions i.e. sunlight, shadow and room. The absolute distance between the lens of the camera and the center of the leaf could be slightly different for each setting because the plant and camera mount were displaced for each condition. The leaf was not held in place by any device and best efforts were made to capture data with no leaf perturbation due to wind. The camera was mounted on a tripod looking down between 45-60 degrees with the horizontal. Orientation of the camera was kept roughly orthonormal (leaf surface normal coincides with Z-axis of the camera). Only a single leaf was in view. On the border of the image, the amplitude is usually lower than in the middle [15], so for simplicity, the entire analysis was done inside a small window (20x20 pixel Region of Interest: ROI) near the center of the image (Fig. 2(b)) which reduces chances of errors in ToF data.

During the test, company provided calibration was used, although the camera was operated at a range shorter than the calibrated range. Calibration only affects the accuracy and not the integrity or precision of the data, and therefore, the depth will not be accurate in meters. This is irrelevant as long as we evaluate pixel values relative to their neighborhood and take into account the validity of data for every pixel under consideration. After all, the purpose of the test is to find an optimal IT, so to calibrate the camera at that IT for higher accuracy.

\section{DATA INTERPRETATION AND METRICS FOR EVALUATION}

CamBoard data is high frame rate (up to $60 \mathrm{fps}$ ), so we analyze it by accumulating it across several frames (Fig. 2(a)) using various statistical measures. As mentioned in Sec. IV, these metrics are estimated in the ROI on the surface of the leaf. The leaf is assumed to be static. In order to empirically estimate the precision (Eq. 5), we first find the mean value of depth for each pixel $i$ across $f$ frames:

$$
\mu_{i}=\frac{\sum_{t=1}^{f} L_{i, t}}{f},
$$



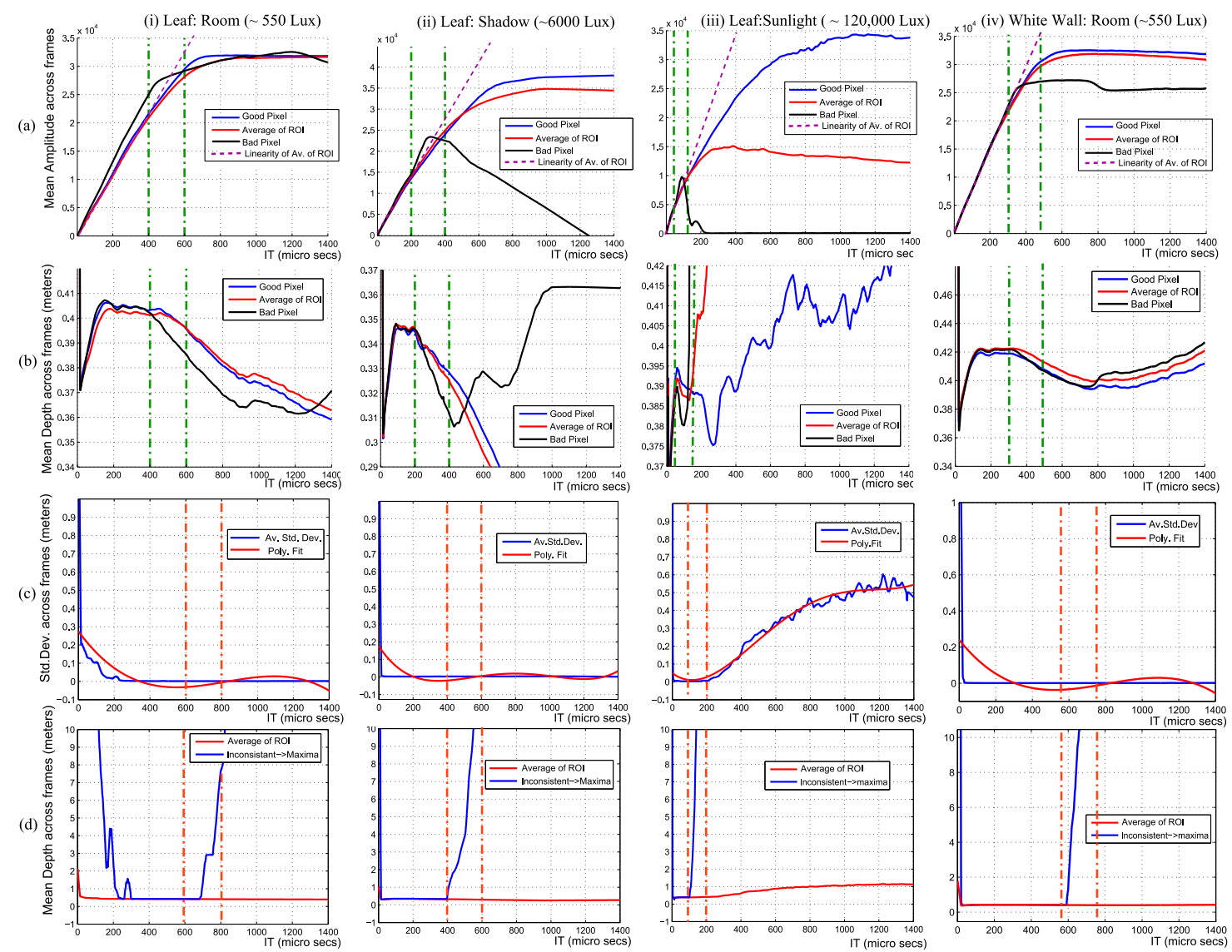

Fig. 3. Analysis of amplitude and depth at $30-35 \mathrm{~cm}$ in a $20 \times 20$ (ROI) under room, full shadow and sunlight conditions for one leaf in view (Fig. 2(b)) and a white wall. A Good pixel is one which does not get marked inconsistent (Tab. I) throughout the IT range while a Bad pixel is one of the first pixels to become inconsistent and hence invalid. Both pixels are inside the ROI (c) Poly. Fit is the trend line for the mean Std.Dev. curve (blue line) of ROI (d) Inconsistent $\rightarrow$ Maxima shows the curve when inconsistent pixels are set to maximum depth manually (a,b) A quick glance shows CamBoard performance in descending order as (iv) best to (i) better, (ii) satisfactory and (iii) worst.

where $L_{i, t}$ is the depth value of pixel $i$ of $t$ frame. The scheme of data accumulation across frame is shown in Fig. 2(a). The standard deviation across frames is:

$$
\sigma_{L_{i}}=\sqrt{\frac{\sum_{t=1}^{f}\left(L_{i, t}-\mu_{i}\right)^{2}}{f-1}} .
$$

In order to extract a more tangible 1D metric to compare precision over IT, we find mean of $\sigma_{L_{i}}$ :

$$
\mu_{\sigma_{L}}=\frac{\sum_{i=1}^{n}\left(\sigma_{L_{i}}\right)}{n}
$$

where $n=N \mathrm{x} N$ is the total number of pixels in the ROI. In the next section, a comparison of these metrics will be done for selected pixels as well as the entire ROI in order to understand the deterioration of depth behavior with increasing IT.

\section{ANALYZING THE EFFECT OF AMBIENT ILLUMINATION ON TOF DATA FOR LEAF IMAGING}

A pixel's amplitude represents the number of photons received by it. Increasing IT linearly increases amplitude.
After a certain point, this behavior becomes non-linear. This is an indication that the pixel is reaching a saturation point where it cannot accommodate incoming photons any further. Saturation, therefore, should be avoided as it renders incorrect depth measurements. May et al. [12] show the over-saturation gap when the mean amplitude curve vs. IT deviates from linearity. We go a step further and find out the reason for this non-linearity. Fig. 3(a,i),(a,ii),(a,iii) show a similar situation for room, shadow and sunlight conditions, respectively. These figures show the mean amplitude across frames for a single pixels and average of the 20x20 ROI on the leaf (Fig. 2(b)) of a scene having one leaf in the view.

In these graphs, good pixels are those that reach saturation amplitude (greater than $3 \times 10^{4}$ ). Contrarily, bad pixels exhibit a very unpredictable behavior and do not necessarily reach the saturation amplitude at all. Due to these bad pixels, the average of ROI deviates from linearity much earlier. Assuming the same material properties for all the pixels on the sensor of the camera, it appears that non-linearity is not related to saturation amplitude only. Fig. 3(b,i),(b,ii)(b,iii) show the corresponding depth values of pixels which start getting out of synchronization as soon as their amplitudes deviate from linearity. The IT 
range where this behavior starts is shown between dotted green bars, we call it the green zone. This is the region providing best trade-off between precision (highest possible amplitude) and depth synchronization among neighboring pixels.

According to May et al. [12] and also by our observation, the deviation from linearity depends on the object distance. For closer objects, it occurs earlier (lesser IT) than for distant objects. It can be seen that ambient illumination affects linearity as well. Deviation occurs earlier under sunlight than in shadow and room conditions. If the IT is increased further than the green zone, the deviation continues and worsens to a point that the PMD onboard system sets inconsistent flag which in-turn also sets the invalid flag (Tab. I). Inconsistency, according to PMD documentation, means multiple depth values pertaining to one pixel or incompatible amplitudes such as saturation. In Fig. 3(a,b), pixels shown as bad become inconsistent at some point, while good pixels do not.

As discussed in section III, depth precision is directly related to the amplitude of the received signal, therefore amplitude must be high enough to enable correct depth measurement, still below the saturation level. Amplitude increases with IT. In order to find the highest possible IT suitable for a given setting, let us consider the standard deviation in Fig. $3(\mathrm{c}, \mathrm{d})$. The mean standard deviation is the mean precision of the ROI (Eq. 8).

As expected, the precision is quite low at very low IT. It improves with increasing IT, but the most important part is the first valley of the trend line i.e. when precision is the highest for the first time. This indicates that there is a consensus among the values of one pixel across frames. Any second valley will not be important because it will be either due to data discontinuity or saturation, the latter would still bring consensus among frames.

The rise of the trend line after the first valley indicates fall of precision which is indicated between red dotted bars, we call it the red zone. Bad pixels start getting marked inconsistent somewhere in the red zone. But the depth or amplitude values may not significantly change at this stage. They are just different from frame to frame, which means precision starts falling. In order to see the corresponding change in the mean depth curve, we set the depth values of the inconsistent pixels to a very high value as soon as the flag is set. The result is displayed in Fig. 3(d). The sharp rise in the curve (blue line) indicates the IT at which the pixels are becoming inconsistent. This provides us the upper threshold of the IT.

In our experiments, the number of inconsistent pixels become significant above approximately 800,600 and $120 \mu$ s for room, shadow and sunlight conditions respectively. The point where the green zone starts usually lies $20-30 \%$ below these values. In other words, we consider appearance of inconsistent flag an early warning of non-linearity of amplitude which could be due to forthcoming saturation or any incompatibility of amplitude and hence increasing IT any further would only worsen the credibility of data.

In all the three conditions, the IT associated with the highest precision lies in the green zone. The ambient light intensity

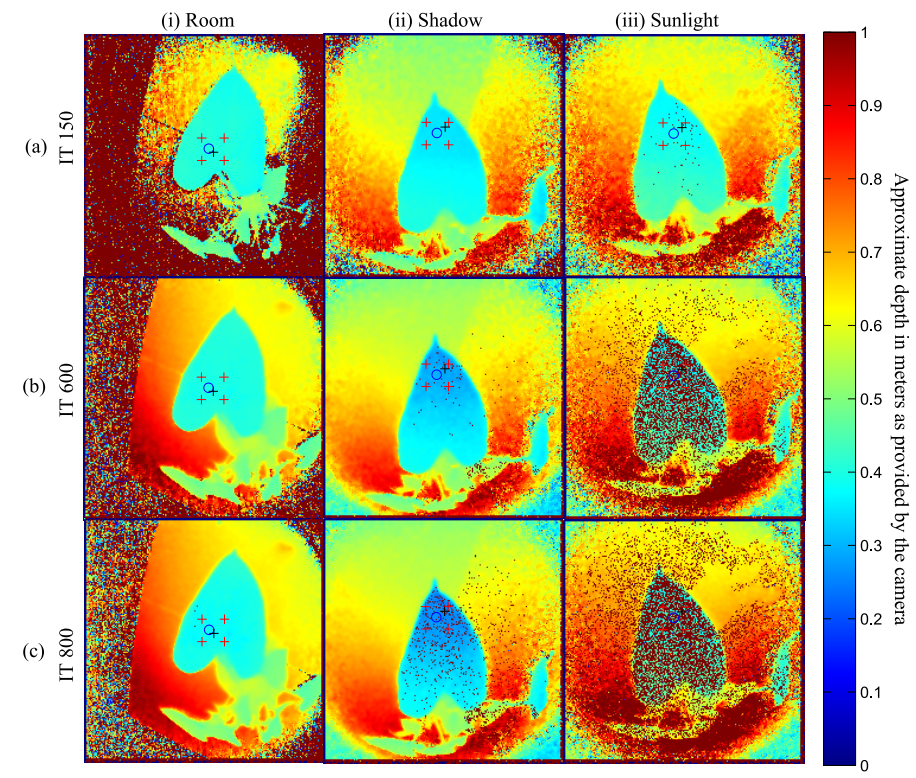

Fig. 4. ToF images of plant leaves under the three different ambient illumination conditions with inconsistent pixels marked maxima, therefore they appear as dark spots on the leaf surface. Red cross indicate ROI, location of a good pixel is indicated by blue circle and a bad pixel by black cross

can vary which will displace the zones slightly over IT. Still, the inconsistent flag serves as an indicator of pixels getting out of synchronization. Fig. 4 shows a sample set of leaf images for the three conditions (columns). The three ITs (rows) are those at which inconsistent pixels are noticeable at any one of the conditions.

\section{A. Comparison of Leaf and White Wall}

Fig. 3 column (iv) shows the ROI characteristics of white wall at approximately $35 \mathrm{~cm}$ from the lens of the camera in room. White wall was chosen in order to benchmark the ToF imaging for leaves as white wall is a highly, if not perfectly, reflecting planar surface and is usually used for testing the ToF imaging quality [15]. We compare it to the best characteristics of the leaf images i.e. room conditions (Fig. 3 column (i)).

Although the reflectivity of the wall was not known, still, it can be assumed to be fairly lambertian with low absorption and transmittance. Wall's imaging characteristics are better than that of the leaf as the amplitude (Fig. 3(a,iv)) and depth (Fig. 3(b,iv)) are more synchronized over the entire range of IT than the leaf. As already discussed in section I-B leaves have high transmittance in NIR. This could possibly be the cause of a slight late appearance of inconsistency whereas the wall has much higher reflectance than transmittance or absorption, so it is more likely to produce saturation earlier. Overall performance of the leaf in the room condition is quite comparable to that of the white wall. This further validates the use of ToF imaging for leaf analysis.

\section{B. Image Enhancement Under Mixed Conditions}

In outdoor applications, it is highly likely to encounter a situation in which a leaf is partly under sunlight and partly 
under shadow. The shadow in such a case would be brighter than a complete shadow due to possible diffraction of sunlight which means that the pixels will become inconsistent a little earlier than shown in Fig. 3(d,ii). Fig. 5(a) and Fig. 5(e) show depth images taken under such a condition at IT 70 and 300 $\mu$ s respectively. These two values are chosen because the flags start appearing at almost $30 \%$ higher IT values than these. Although Fig 5(c) and Fig. 5(d) show no pixels on the leaf flagged at IT $70 \mu \mathrm{s}$, but the part of leaf under shadow will be having low precision. Therefore, 70 and $300 \mu$ s ensure operation in green zone for both exposures hence providing high precision. In order to improve the depth data, we replace the depth values of all the pixels with Inconsistent and Low Intensity flags set at IT $300 \mu$ s with the corresponding values from IT $70 \mu \mathrm{s}$. The result is shown in Fig. 5(i). With this approach we will only require calibration for the two IT values and as discussed in section VI, this will enable us to achieve both high precision and optimal accuracy. The reason for including Low Intensity flags as well is due to the fact that we have noticed that some of the pixels receiving multiple reflections may also be flagged as low signal, perhaps due to accepting lower amplitude among the multiple values.

\section{CONCLUSION}

We have tested CamBoard under sunlight, shadow and room conditions and presented a detailed analysis of its behavior for the particular case of close range plant imaging. Comparison with flat white wall indicate suitability of using ToF imaging in agricultural applications. As ToF cameras are sensitive to ambient light, we have proposed a method to find a suitable IT for the three conditions using the appearance of inconsistent flags in PMD devices. This scheme can be extended to any ambient illumination setting. Choosing a specific IT for a given condition allows higher accuracy through optimal calibration for that IT, which adds to the value of imaging in close range tasks such as plant phenotyping in which robot grasping tasks may be involved. Although the results presented in this article are only limited to one type of plant, they are still sufficient to validate the approach. While the corresponding camera settings may change slightly with the plant species and leaf thickness, this work provides a method to find optimal ToF camera parameters for any plant, ambient illumination and range.

\section{ACKNOWLEDGEDMENTS}

Authors want to thank PMDTec and Ilona Schwengber for their support and insightful information about CamBoard.

\section{REFERENCES}

[1] R. Lange and P. Seitz, "Solid-state time-of-flight range camera," Quantum Electronics, IEEE Journal of, vol. 37, no. 3, pp. 390-397, 2001.

[2] S. Foix and G. Alenya, "Lock-in time-of-flight (ToF) cameras: a survey," Sensors Journal, IEEE, vol. 11, no. 9, pp. 1917-1926, 2011.

[3] F. Mufti and R. Mahony, "Statistical analysis of signal measurement in time-of-flight cameras," ISPRS Journal of Photogrammetry and Remote Sensing, vol. 66, no. 5, pp. 720-731, Sep. 2011.

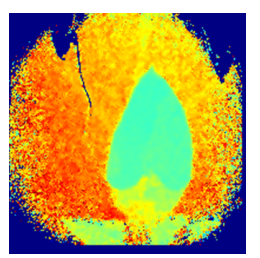

(a) IT 70 Depth

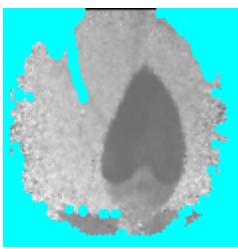

(d) Low Intensity

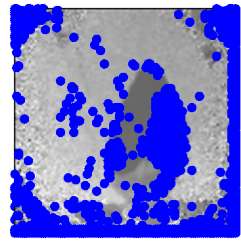

(g) Inconsistent

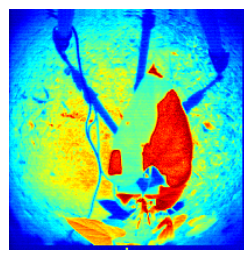

(b) Intensity

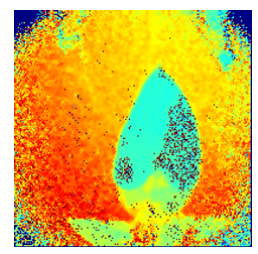

(e) IT 300 Depth

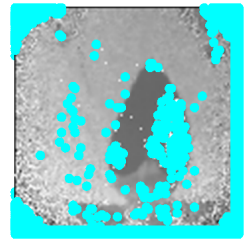

(h) Low Intensity

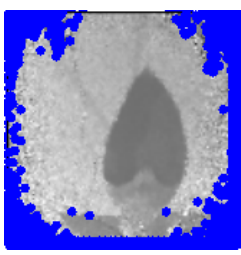

(c) Inconsistent

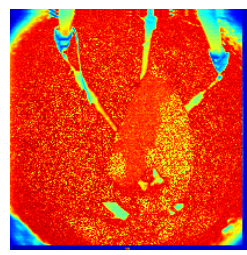

(f) Intensity

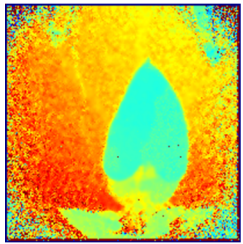

(i) Combined Depth
Fig. 5. Leaf depth and intensity images (in jet color scheme) under mixed conditions and corresponding flagged pixels (Low Intensity pixels in cyan and Inconsistent pixels in blue). (a,b,c,d) at IT 70 and $(e, f, g, h)$ at IT 300

[4] R. Lange, "3D time-of-flight distance measurement with custom solidstate image sensors in CMOS/CCD-technology," Ph.D. Dissertation, University of Siegen, 2000.

[5] W. Kazmi, M. Bisgaard, F. Garcia-Ruiz, K. Hansen, and A. la CourHarbo, "Adaptive Surveying and Early Treatment of Crops with a Team of Autonomous Vehicles," in European Conference on Mobile Robots, Orebro, Sweden, 2010, pp. 253-258.

[6] G. Alenya, B. Dellen, and C. Torras, "3D modelling of leaves from color and ToF data for robotized plant measuring," in IEEE International Conference on Robotics and Automation, Shanghai, China, 2011, pp. 3408-3414.

[7] R. Klose, J. Penlington, and A. Ruckelshausen, "Usability study of $3 \mathrm{~d}$ time-of-flight cameras for automatic plant phenotyping," Bornimer Agrartechnische Berichte, vol. 69, pp. 93-105, 2009.

[8] M. Kraft, N. Regina, S. a. D. Freitas, and A. Munack, "Test of a 3D Time of Flight Camera for Shape Measurements of Plants," in CIGR Workshop on Image Analysis in Agriculture, no. August, Budapest, 2010.

[9] A. D. Nakarmi and L. Tang, "Inter-plant Spacing Sensing at Early Growth Stages Using a Time-of-Flight of Light Based 3D Vision Sensor," in ASABE Paper No. 1009216, St. Josheph, Michigan, 2010, pp. $1-15$.

[10] J. T. Woolley, "Reflectance and transmittance of light by leaves." Plant physiology, vol. 47, no. 5, pp. 656-62, May 1971.

[11] S. Jacquemoud and F. Baret, "PROSPECT: A model of leaf optical properties spectra," Remote sensing of environment, vol. 34, no. 2, pp. 75-91, Nov. 1990.

[12] S. May, B. Werner, and H. Surmann, "3D time-of-flight cameras for mobile robotics," in Intelligent Robots and Systems, IEEE International Conference on, Beijing, China, 2006, pp. 790- 795.

[13] “Mesa Imaging," http://www.mesa-imaging.ch.

[14] "PMDTec," http://www.pmdtec.com.

[15] T. Kahlmann and F. Remondino, "Calibration for increased accuracy of the range imaging camera SwissRanger," in ISPRS Commission V Symposium, no. 4, Dresden, Germany, 2006, pp. 136-141. 